TP Periodica Polytechnica Mechanical Engineering

\author{
62(3), pp. 179-186, 2018 \\ https://doi.org/10.3311/PPme.11088 \\ Creative Commons Attribution (i)
}

RESEARCH ARTICLE

\section{Influence of Rotational Wall of Axial Inlet Device on Velocity Distribution at Impeller Inlet}

\author{
Oleksandr Moloshnyi ${ }^{1 *}$, Mykola Sotnyk ${ }^{1}$, Svitlana Lugova $^{2}$
}

Received 30 May 2017; accepted after revision 15 May 2018

\begin{abstract}
The goal of the article is to identify the influence of geometrical parameters of the axial inlet device, presence of its walls rotation and the pump flow rate on components of the absolute velocity distribution at the impeller inlet.

A numerical simulation in the software ANSYS CFX and analysis of liquid flow structure of a double entry centrifugal pump were carried out. The axial inlet device is shaped by cylindrical and diffuser section, which includes fairing in front of the impeller. Four models of the axial inlet casing were researched.

In the analyzed variants of geometry of the axial inlet device, structure of absolute velocity distribution component is similar. Rotational speed of $1450 \mathrm{rpm}$ of the rotational walls of the axial inlet device compared to fixed walls affects the change of distribution of an axial component of the absolute velocity more than circumferential. Introduction of sudden expansion at the impeller inlet reduces the average value of the circumferential velocity and improves the axial velocity components distribution. Maximum and average value of the axial component of the absolute velocity and its unevenness in cross-section of the intake reduces while reducing the pump flow rate.
\end{abstract}

\section{Keywords}

centrifugal pump, inlet nozzle, suction casing, inlet flow structure, $C F D$

\footnotetext{
${ }^{1}$ Applied Hydro- and Aeromechanics Department,

Faculty of Technical Systems and Energy Efficient Technologies,

Sumy State University,

40007 Sumy, Rymsky-Korsakov St., 2, Ukraine

2 JSC "VNIIAEN", 40003 Sumy, 2-ya Zheleznodorozhnaya St., 2, Ukraine

*Corresponding author, e-mail: o.moloshnij@pgm.sumdu.edu.ua
}

\section{Introduction}

A pump design provides an optimal configuration of its flowing part to provide necessary operating parameters with the highest possible level of efficiency. One of the main elements of a pump, which affects the efficiency of its operating process, is inlet casing. Its design affects the value of hydraulic losses and forms a flow structure at the outlet of inlet casing. Its uneven distribution in a cross-section at the impeller inlet leads to various directions of fluid along blades leading edge. As a result, there is an uneven distribution of fluid pressure at the impeller inlet, which affects the formation of vortices. This impairs efficiency and cavitation performance of the pump and causes vibration [1].

Many scientists conducted researches of a structure of fluid flow at the impeller inlet. There are quite interesting works of some authors. Karapuzova [2] analyzed various designs of combined semi-volute inlet casing and determined that the circumferential component of the absolute velocity significantly increases on the periphery of a cross-section at the impeller inlet but axial reduces. Elin et al. [3] explored distribution of the velocities and pressures at the impeller inlet. The authors obtained good quantitative and qualitative convergence results of the numerical simulation of the flow structure at the inlet casing with experimental data. Lipej and Mitrushevski [4] analyzed the liquid flow structure in the inlet casing in the double entry centrifugal pump in the range of patrload operation. The authors investigated an uneven distribution of the axial component of the absolute velocity at the impeller inlet. Type (cross-sectional shape) of inlet casing also affects the flow structure. Song et al. [5] discovered that flat inlet elbow forms a less circumferential component of absolute velocity of a flow compared to asymmetric inlet casing and the large radius elbow. Rudolf and Klas [6] studied a vortex flow structure at the inlet of the vertical submersible pump, which pumped fluid from open water, and found that the uneven absolute velocity distribution increases with rise of the flow rate.

Stepanof [7] considers that the best option of the inlet device is a straight axial converging tube. It stabilizes the fluid flow and provides its delivery with a uniform axial component of 
the absolute velocity in the flow cross-section. The flow structure in such an inlet casing in the overhung single stage pumps is analyzed in articles [8-11]. The authors found little uneven distribution of components of the absolute velocity with an increasing radius of a flow cross-section. Zhang et al. [12] discovered a swirling liquid flow structure in the whole volume of an inlet casing, the direction of which coincides with the direction of rotation impeller. Lei et al. [13] investigated influence of guide blades on a flow structure. A positive pre-whirl reduces the area of reverse flow and uneven distribution of the absolute velocity components. A negative pre-whirl increases the area of the reverse flow and uneven distribution of the absolute velocity components.

Analysis of the literature shows that little attention is paid to the analysis of the liquid flow structure in the axial inlet device with diffuser section. However, Rosa and Pinho [14] rated pressure loss coefficient in axially symmetric diffuser Csizmadia and Hos [15] determined loss coefficient of friction for the Non-Newtonian fluids that flowed through the diffuser.

Most reviewed papers analyzed the influence of shape or size of the inlet device on the flow structure, including the direction and magnitude of the circumferential and axial components of the absolute velocity. However, they do not show the flow structure dependence on the rotation of the inlet device walls. Consideration of the influence of this factor on the liquid flow structure at the impeller inlet device could improve operating process of pump. Consequently, the life cycle cost of the pump will be reduced. Thus, the main goal of the work is to identify: the influence of the geometrical parameters of the axial inlet device, rotating speed of the inlet walls and the change of the pump flow rate on the components of the absolute velocity distribution at the impeller inlet.

\section{Theoretical overview}

Theoretical head of a pump impeller is calculated with a well-known mathematical expression [1]. That takes into account the circumferential velocity of the impeller, the relative velocity of the fluid flow in the impeller and absolute flow velocity. At the impeller inlet, the latter can be represented by the following components: circumferential $\left(V_{1 u}\right)$, axial $\left(V_{1 a}\right)$ and radial $\left(V_{l r}\right)$ (Fig. 1). Their size and direction affect the quantity of a head. More often, for determining the theoretical head Euler equation is used [1], which directly takes into account the influence of circumferential component of absolute velocity on the pump head. In general, the average value of the velocities or their components in the cross section is used to simplify the theoretical and practical calculations [6]. This makes a constant error in calculation results. However, the influence of the inlet device on the flow structure can be estimated by a coefficient of non-uniformity for the field of axial velocity.

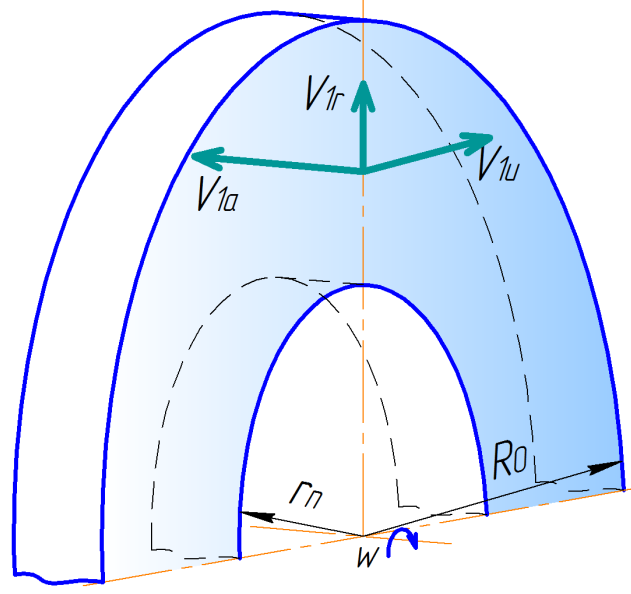

Fig. 1 Composites of absolute velocity of the liquid in the cross-section at the impeller inlet ( $r_{n}$ and $R_{0}-$ hub and impeller eye radius respectively)

The flow swirl factor at the impeller inlet can be defined as [2]:

$$
\mu_{\tau}=\frac{\overline{V_{1 u} \cdot r}}{Q} \cdot D_{q}
$$

where $\overline{V_{1 u} \cdot r}$ is the averaged velocity moment in the plane;

$D_{q}$ is the equivalent diameter of the impeller inlet:

$$
D_{q}=\sqrt{D_{0}^{2}-d_{n}^{2}}
$$

$Q$ is the flow rate.

The coefficient of non-uniformity for the field of the axial velocity [2]:

$$
\chi=\frac{2 K_{n}}{\rho \cdot \overline{V_{1 a}^{2}}}
$$

where $K_{n}$ is the average kinetic energy of $1 \mathrm{~kg}$ of liquid at the outlet section of the inlet casing for the axial velocity component:

$$
K_{n}=\frac{1}{Q} \cdot \int_{F} \frac{\rho \cdot \overline{V_{1 a}^{3}}}{2} d F
$$

$\rho$ is the fluid density;

$\overline{V_{1 a}}$ is the averaged axial absolute velocity component in the cross-section;

$F$ is the cross-sectional area.

\section{Materials and methods}

To determine the liquid flow structure in the axial inlet device of the double entry centrifugal pump the numerical simulation of the operating process was made. The pump parameters were design flow rate $Q_{\text {nom }}=25 \mathrm{~m}^{3} / \mathrm{h}$, design head $H=12$ $\mathrm{m}$, rotational speed $n=1450 \mathrm{rpm}$, specific speed $n_{q}=13$, impeller outer diameter $D_{2}=0.202 \mathrm{~m}$.

The pump axial inlet device is simultaneously the hollow shaft of the hermetic canned motor pump. The inlet device is shaped as a cylindrical and diffuser section in front of the impeller, which includes fairing (Fig. 2a). 


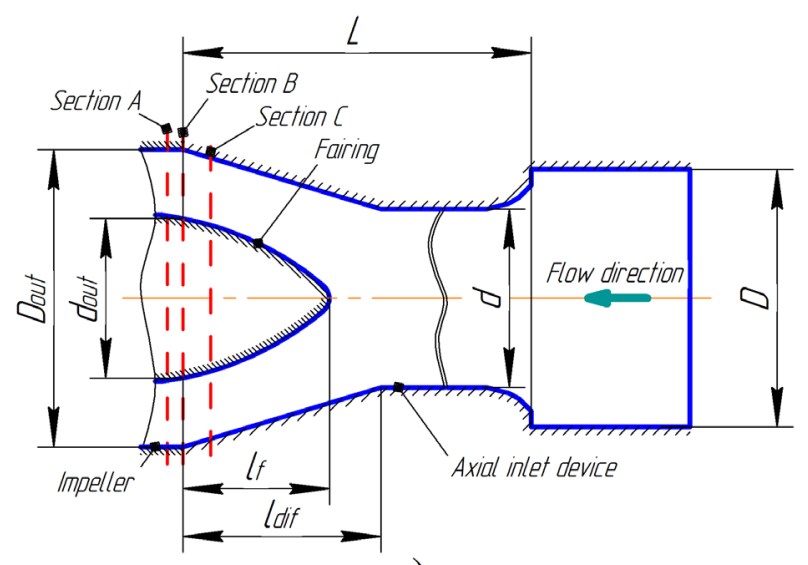

a)

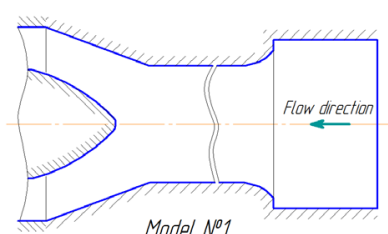

Model No1
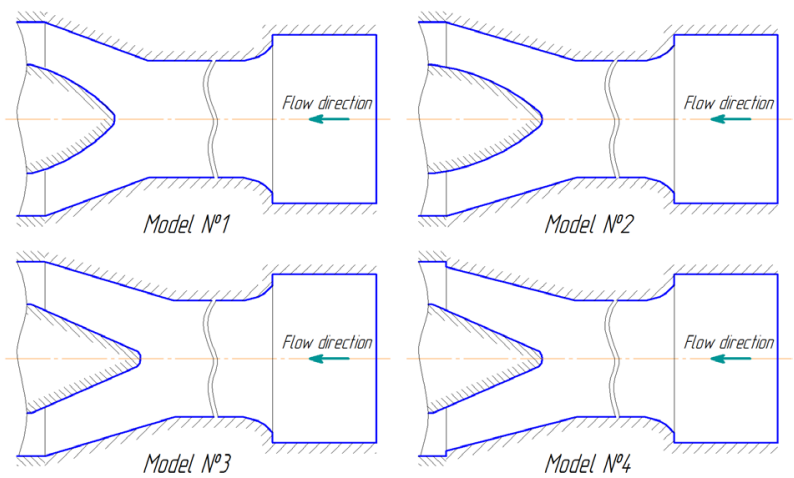

b)

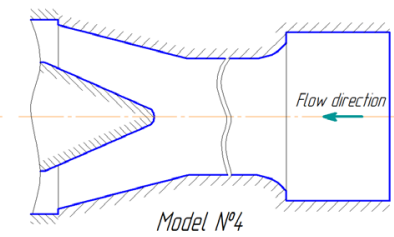

Fig. 2 Scheme of the axial inlet device:

a) geometric parameters; b) models schemes

The numerical simulation of the fluid flow was conducted for 4 axial inlet device models (Fig. 2b). The diameter of the cylindrical section $d$ and the inlet $D$ are $45 \mathrm{~mm}$ and $65 \mathrm{~mm}$ respectively. The length of rotational wall of the axial inlet device is $L$ $=227 \mathrm{~mm}$. The impeller eye diameter is $D_{0}=75 \mathrm{~mm}$. The length of the fairing $\left(l_{f}\right)$ and the diffuser section of the axial inlet device $\left(l_{d i f}\right)$, external $\left(D_{\text {out }}\right)$ and internal $\left(d_{\text {out }}\right)$ diameter of the outlet of the diffuser section are shown in Table 1. Axial dimensions of the pump housing limit the inlet device dimensions.

A solid model of a fluid computational domain of the double entry centrifugal pump includes the inlet pipe, the outlet pipe, the axial inlet device, the impeller and the annular casing with guide vanes (Fig. 3a). The numerical model was simplified by assumption of neglecting of the leakage between the front shroud and the pump casing. Also, symmetry flow relative to the impeller of the double entry centrifugal pump was considered. This reduces complexity and increases the speed of calculation.

Table 1 Geometrical parameters of the axial inlet device

\begin{tabular}{lcccc}
\hline & \multicolumn{4}{c}{ Model } \\
\cline { 2 - 5 } & №1 & №2 & №3 & №4 \\
\hline $\mathrm{D}_{\text {out }}(\mathrm{mm})$ & 75 & 75 & 75 & 71 \\
$\mathrm{~d}_{\text {out }}(\mathrm{mm})$ & 41 & 41 & 40 & 40 \\
$1_{\text {dif }}(\mathrm{mm})$ & 40 & 50 & 50 & 50 \\
$1_{\mathrm{f}}(\mathrm{mm})$ & 27 & 37 & 37 & 37 \\
\hline
\end{tabular}
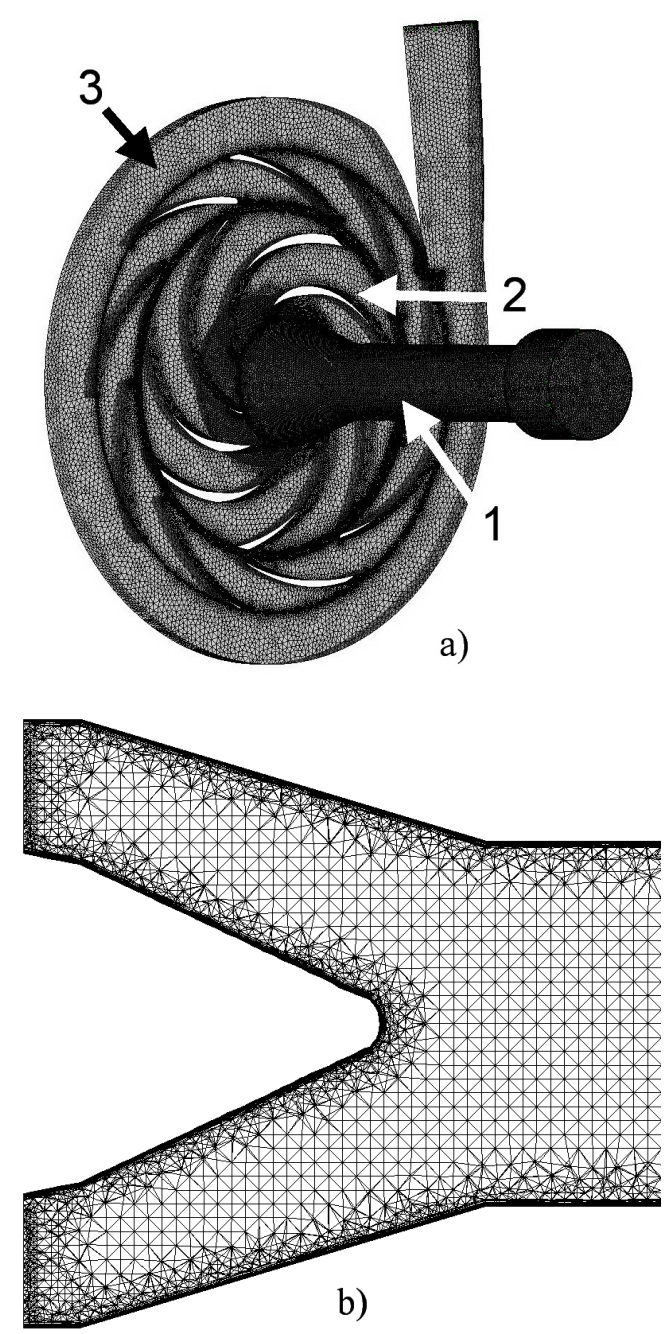

Fig. 3 Example of the mesh: a) calculation area of pump flowing part: 1 axial inlet device, 2 - impeller, 3 - annular casing; b) diffuser section of the axial inlet device fluid domain

An unstructured mesh was generated using software ICEMCFD (Fig. 3). Elements size was selected via mesh independence research. Layers of prismatic elements were created near solid walls in the boundary layer, (Fig. 3b). The total number of elements of the pump flowing part is 4.4 million. The inlet device, the impeller and the annular casing contain $0.97 \mathrm{mln}$, $1.76 \mathrm{mln}$ and $1.67 \mathrm{mln}$ of elements respectively.

The numerical simulation of the fluid flow in the flowing part of the pump was conducted using the software ANSYS CFX at the stationary setting and with the standard $k-\varepsilon$ turbulence model. Boundary conditions were set as inlet -the mass flow rate, output - the static pressure. The numerical calculations were carried out for a range of the flow rate $(0.6-1.2) Q_{\text {nom }}$. Working fluid was water at temperature $25^{\circ} \mathrm{C}$. The roughness of the inlet device surfaces adopted is 6.3 microns. The value of $Y+$ for solid walls is below 60 , which is suitable for the chosen model of turbulence.

\section{Numerical simulation and analysis}

The results of the numerical simulation identify the fluid flow structure in the pump axial inlet device. Visualization of 
research results of flow indicates the nature of the distribution of absolute velocity vectors in the longitudinal (Fig. 4) and cross (Fig. 5) sections of axial inlet device.

Near the fairing, the axial component of the absolute velocity is predominant and on the periphery - circumferential component. This is caused by the backflow in the impeller inlet, which is created by the blade angle at the inlet, as well as the diffuser section of the axial inlet device. There is a logical reduction in absolute speed near the walls of the diffuser section and the stagnant zone [1]. In addition, fairing divides the flow in the middle and causes growth of the axial component of the absolute velocity. The impeller and rotary motion of the axial inlet device walls influence the liquid flow swirl in the peripheral zone. Model №4 has less stagnant zone due to a sudden expansion. As a result, the zone of low velocity near the walls of the diffuser section becomes longer.

To compare the absolute velocity distribution of the fluid flow structure three cross-sections $A, B$ and $C$ were selected (Fig. 2a). The distance from the cross section $\mathrm{B}$ to the cross section $A$ and $C$ are $4 \mathrm{~mm}$ and $7 \mathrm{~mm}$ respectively. The cross section $A$ is located in the impeller eye, the cross-section $B$ - at the impeller inlet and the cross section $C$ - at the beginning of the stagnant zone. Analysis of the velocity distribution confirms the significant reduction of the circumferential velocity component in the cross sections with its distance from the impeller (Fig. 5). This is caused by the decrease of the impact of the impeller on the swirl of the flow.

Since the inlet casing geometry is axisymmetric, flow is also considered axisymmetric. This is confirmed by the picture of vectors of absolute velocity distribution in the cross sections (Fig. 5). Therefore, analysis of varying the absolute velocity components is conducted along one of the positions of the selected radius in the cross-sections.

The analysis of averages of the absolute velocity components along the radius shows little alteration, which depends on the change of the axial intake geometric dimensions (Table 2). Introduction of the sudden expansion in the model №4 is causing reduction circumferential component by $19 \%$ and growth axial by $2.6 \%$. In addition, the average and maximum values of the radial component of absolute velocity are much lower than the other two components.

To compare the distribution of absolute velocity components the diagrams in the different cross sections were prepared (Fig. 6-9). For ease of comparison, the value of velocity components and radii were presented in relative terms according to their average $\left(V_{\text {(ave) }}\right)$ and maximum $\left(R_{\max }\right)$ value in the relevant cross-section. Also, all cross-sectional areas are divided into two circular zones: zone 1 covers the area of fluid flow to 0.75 $R_{\max }$ and zone 2 covers the area in the range $(0.75-1) R_{\max }$.

Generally, the distribution of absolute velocity components is similar in comparison to all models at the cross section $B$ (Fig. 6). There are significant differences in the diagrams of

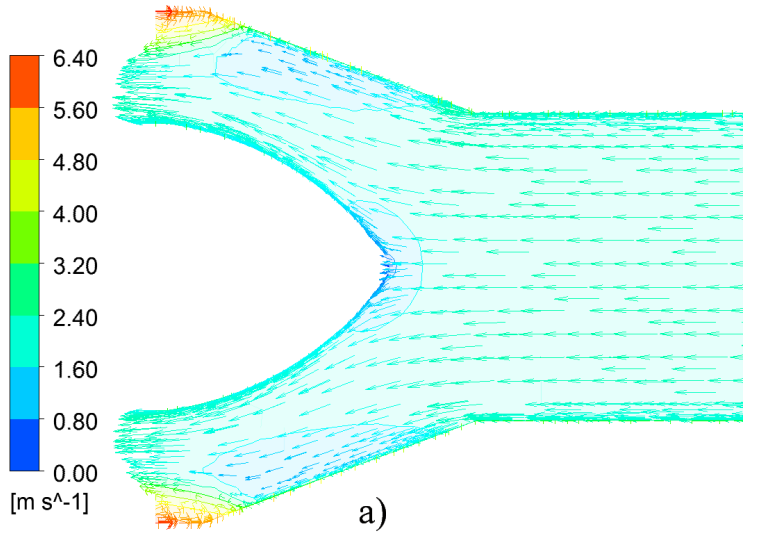

a)
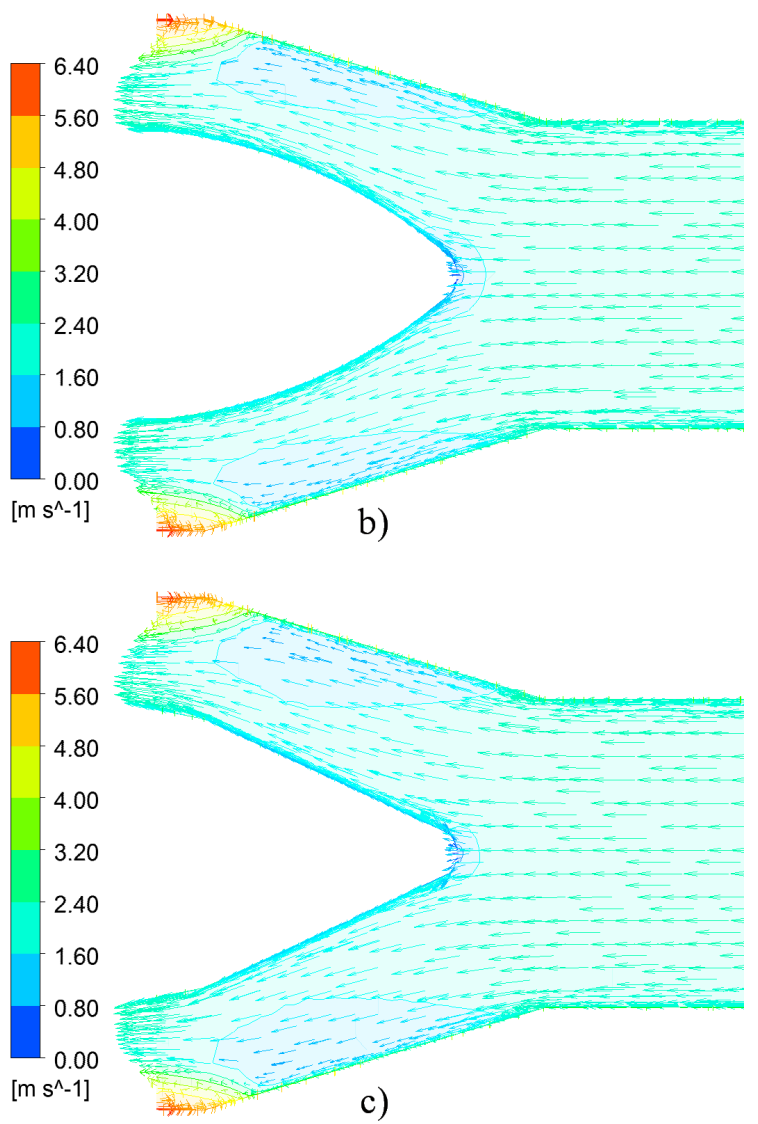

c)

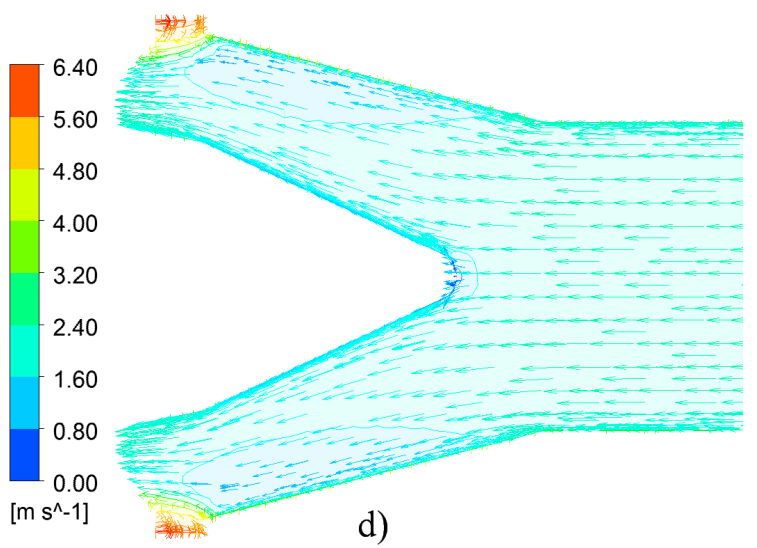

Fig. 4 Absolute velocity distribution at the longitudinal section of the diffuser section of the axial inlet device flow: a) model №1; b) model №2; c) model №3; d) model №4 


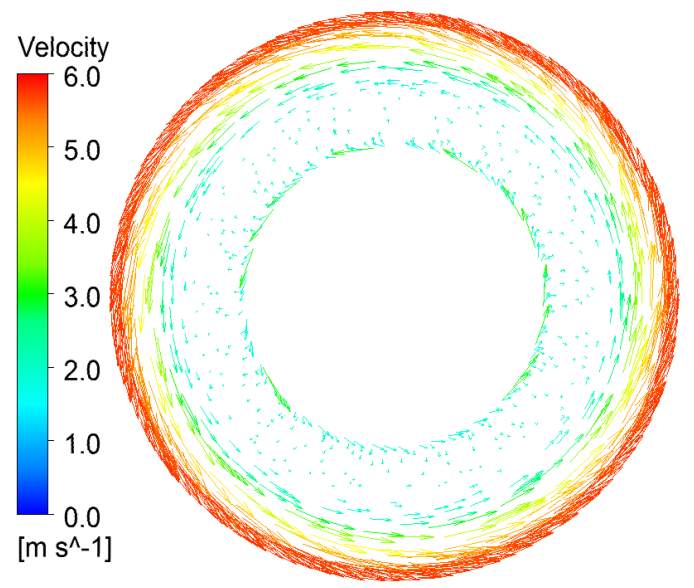

a)

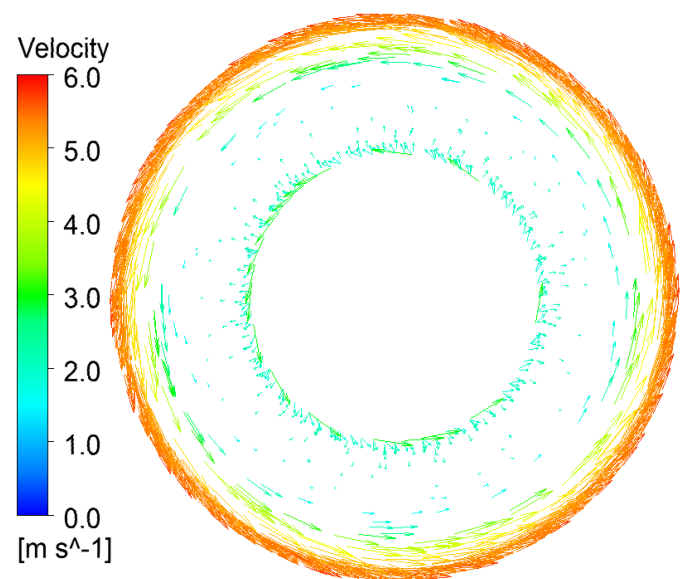

b)

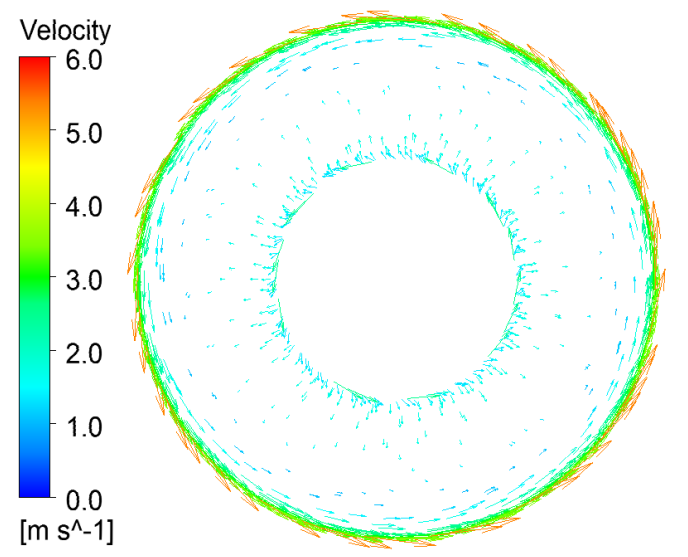

c)

Fig. 5 Absolute velocity distribution of the flow at the model №3 a) cross section $\mathrm{A}$; b) cross-section $\mathrm{B}$; c) cross section $\mathrm{C}$
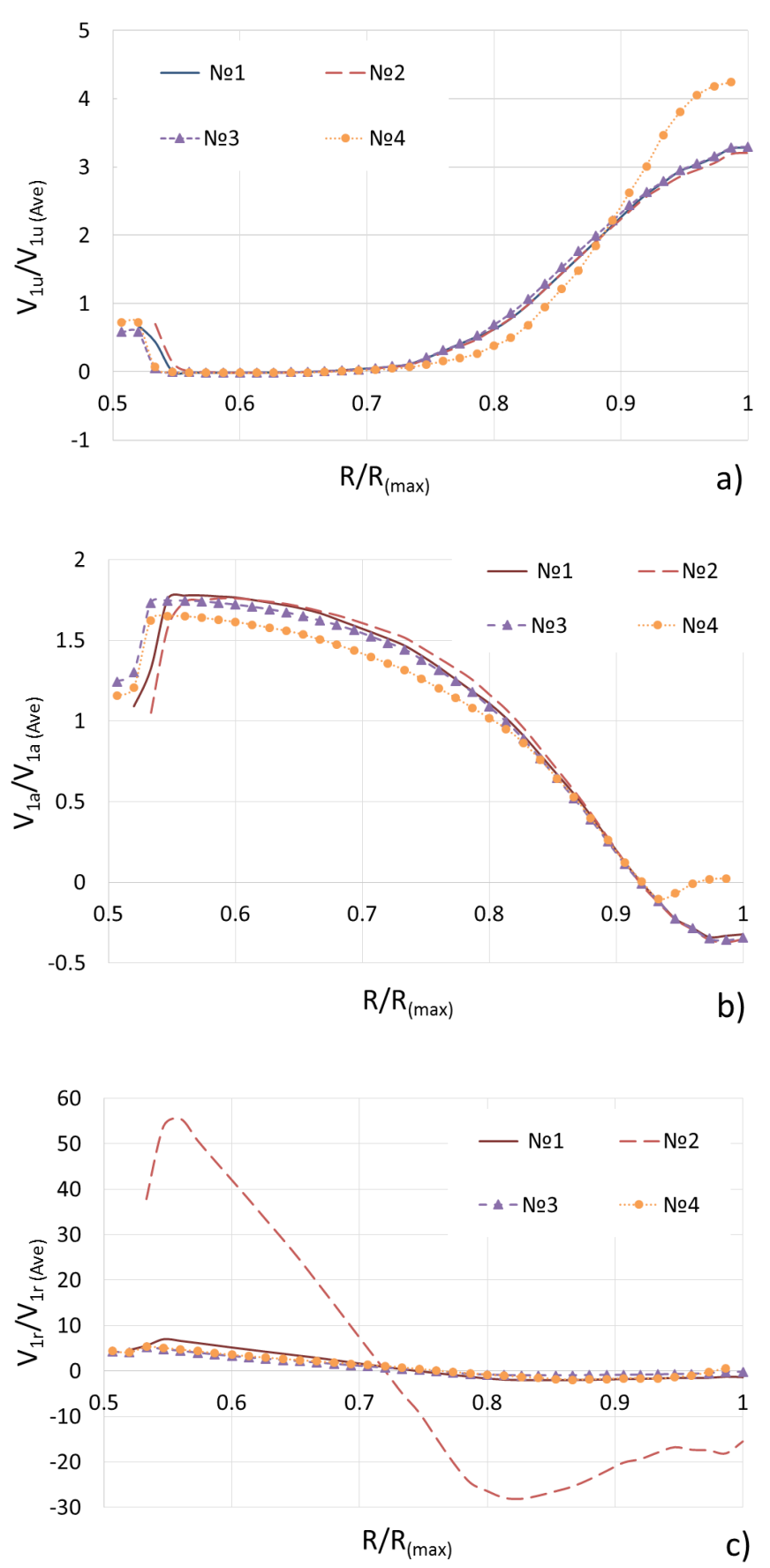

Fig. 6 Diagram of absolute velocity components distribution in the crosssection $B$ at the flow rate $Q_{\text {nom }}$ : a) Circumferential velocity component;

b) Axial velocity component; c) Radial velocity component the model №4 in zone 2 (Fig. 6a). In the range (0.75-0.9) $R_{\max }$ relative magnitude of the circumferential component is $1 / 10$ times smaller than in other models. In the range (0.9-1.0) $R_{\max }$ relative magnitude of the circumferential component is almost 4 times bigger than the average value. The reason for this division is the sudden expansion of the outlet of the axial inlet device that isolates stagnant zone. In this zone on the periphery of the cross-section, there is almost no reverse direction of the fluid (Fig. 6 b). In zone 1 the vectors of the radial component are directed toward the periphery, which is caused by the fairing shape, and in the zone 2 - the direction is opposite, probably due to the rising circumferential component of the absolute velocity and the effect of the wall (Fig. 6 c). A significant increase of the relative value of the radial component of absolute velocity in the model №2 is explained by the low mean value close to zero (Table 2) and the course of the curve should be treated as nocompariable.

The comparison of the diagrams of absolute velocity components distribution in the model №3 in the cross-sections $A$, $B$ and $C$ (Fig. 7) indicates the changing of the flow structure. 
Table 2 Average value of absolute velocity components

\begin{tabular}{lcccc}
\hline & \multicolumn{5}{c}{ Model } \\
\cline { 2 - 5 } Velocity component & 1 & 2 & 3 & 4 \\
\cline { 2 - 5 } & \multicolumn{4}{c}{ Cross section A } \\
\hline Circumferential (m/s) & 1.987 & 2.018 & 1.937 & 1.899 \\
Axial(m/s) & 1.212 & 1.21 & 1.188 & 1.186 \\
Radial(m/s) & 0.032 & 0.011 & 0.073 & 0.033 \\
\hline & & Cross section B \\
\hline Circumferential (m/s) & 1.676 & 1.722 & 1.654 & 1.339 \\
Axial (m/s) & 1.168 & 1.168 & 1.163 & 1.193 \\
Radial (m/s) & 0.07 & 0.006 & 0.124 & 0.114 \\
\hline & & Cross section C \\
\hline Circumferential (m/s) & 0.545 & 0.532 & 0.54 & 0.357 \\
Axial (m/s) & 1.246 & 1.272 & 1.19 & 1.303 \\
Radial (m/s) & 0.308 & 0.174 & 0.26 & 0.378 \\
\hline
\end{tabular}

Velocity distributions in the cross sections $A$ and $B$ are qualitatively the same and quantitatively similar. The distribution component of absolute velocity in the cross-section $C$ depends only on the rotation of the walls of the axial inlet device. Therefore, the average circumferential component of the absolute velocity in this cross section is three times smaller than in other cross-sections. In addition, the direction of the axial component of the absolute velocity is not reversed. The vector of the radial component of the absolute velocity has a preferred direction towards the periphery (Fig. 7c). The reason of this is the presence of fairing.

At the outlet of the diffuser section, fluid is swirled by the impeller and circumferential component of absolute velocity increases similar to the situation described in $[6,11]$. Moreover, the uneven distribution of the absolute velocity vectors increases.

The comparison of the diagrams of absolute velocity components distribution in the models №3 and №4 in the cross-section $B$ in models with rotational and motionless inlet walls (Fig. 8) indicate that distribution is qualitatively similar except the top of zone 2 (region $(0.9-1.0) R_{\max }$ ). In the region $(0.75-$ $0.9) R_{\max }$ of zone 2 , the values of the circumferential component of absolute velocity in the model №4 are smaller than in the model №3 by 10-15\%. It is also explained by the sudden expansion at the outlet of the diffuser section. In addition, the uneven distribution of radial component in zone 2 in the model №4 is observed. The cause of this is changing of the flow direction in the area of the sudden expansion.

The flow swirl factor for the models №1, №2 and №3 in the cross-section $B$ are similar to each other in the model with the rotation inlet walls (Table. 3). Also, it is similar to each other in the case of motionless inlet walls. The rotation inlet walls cause the growth of the flow swirl factors 4-5 times for models №1, №2 and №3. It is $185 \%$ and 12\% bigger for the model №4 than for the model №3 respectively for rotational
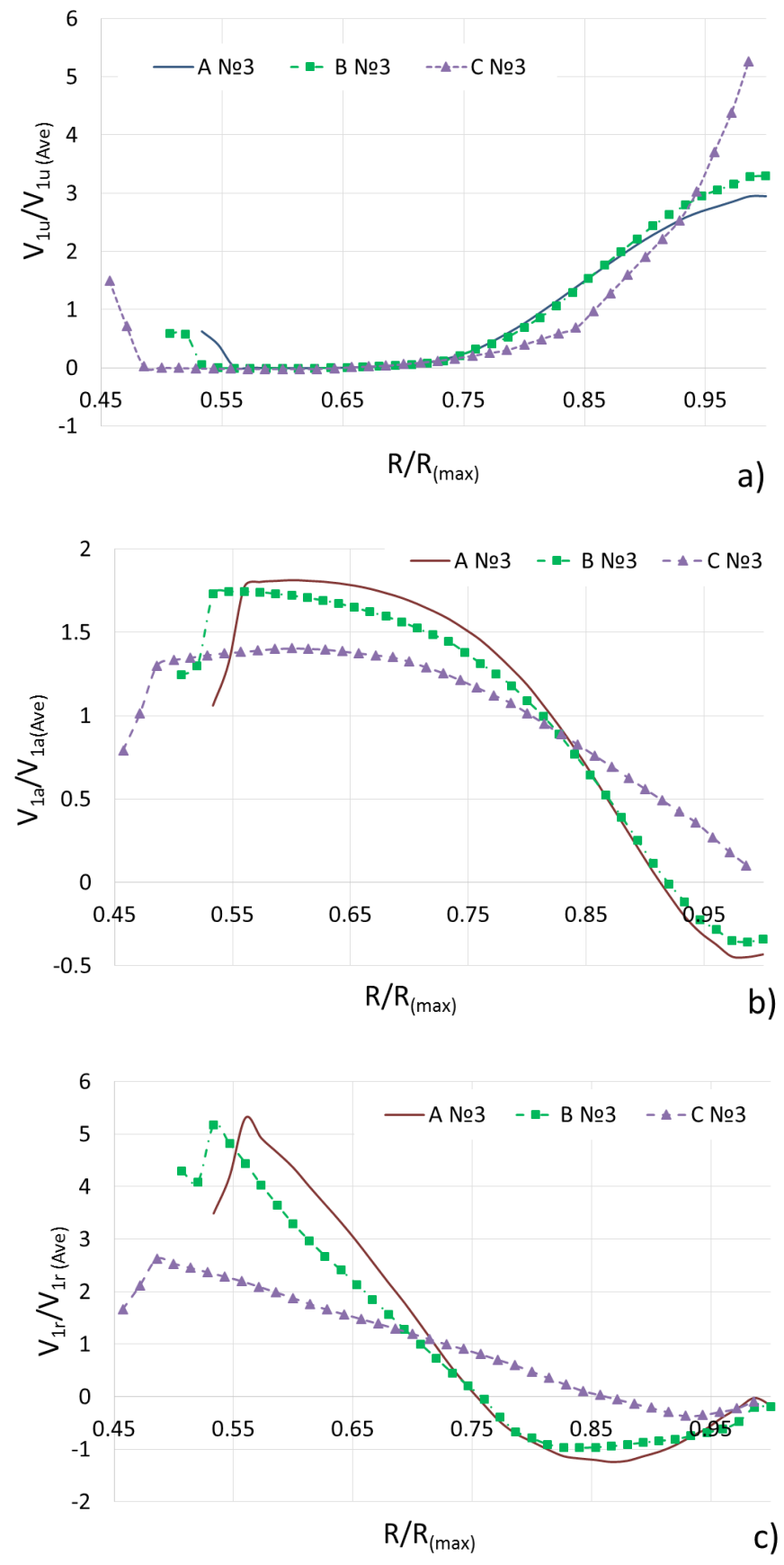

Fig. 7 Diagram of absolute velocity components distribution in the crosssections $A, B$ and $C$ in the model №3 at the flow rate $Q_{\text {nom }}$ :

a) Circumferential velocity component; b) Axial velocity component; c) Radial velocity component

and motionless inlet walls. It could be concluded, that application of the sudden expansion increases the size of the swirl flow at the impeller inlet. This is due to the growth of velocity in the stagnant zone on the periphery. The coefficients of non-uniformity of the field of axial velocity $(\chi)$ for the models №1, №2 and №3 are similar. There is minor influence of walls rotation. The coefficients grow by $8-9 \%$. The model №4 has a much greater coefficient, which characterizes the structure of the flow as more uniform. The axial inlet device with rotational walls causes the growth of $\chi$ factor by $11 \%$. 

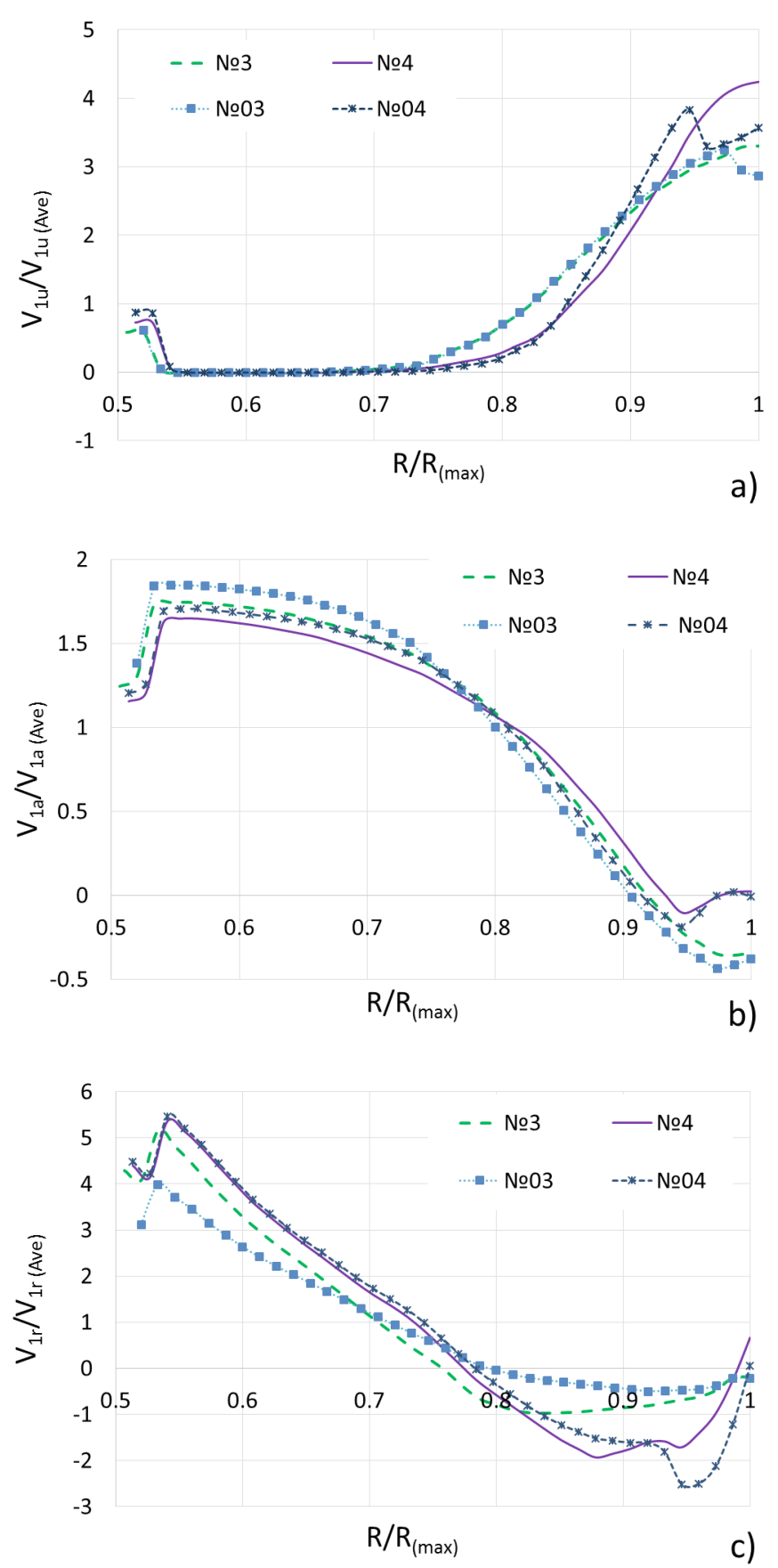

Fig. 8 Diagram of absolute velocity components distribution in the crosssection $B$ in the model №3 and №4 at the rotational speed 1450 rpm (curves №3 and №4) and $0 \mathrm{rpm}$ (curves №03 and №04) at the flow rate $Q_{\text {nom }}$ : a) Circumferential velocity component; b) Axial velocity component; c) Radial velocity component

Table 3 Coefficiens of the inlet device hydraulic performances

\begin{tabular}{ccccc}
\hline № & \multicolumn{2}{l}{ Flow swirl factor $\left(\mu_{\tau}\right)$} & $\begin{array}{r}\text { Coefficient of non-uniformity for } \\
\text { the field of axial velocity }(\chi)\end{array}$ \\
\hline & $0 \mathrm{rpm}$ & $1450 \mathrm{rpm}$ & $0 \mathrm{rpm}$ & $1450 \mathrm{rpm}$ \\
1 & 0.027 & 0.137 & 0.227 & 0.249 \\
2 & 0.033 & 0.146 & 0.205 & 0.222 \\
3 & 0.021 & 0.137 & 0.205 & 0.222 \\
4 & 0.06 & 0.153 & 0.459 & 0.511 \\
\hline
\end{tabular}
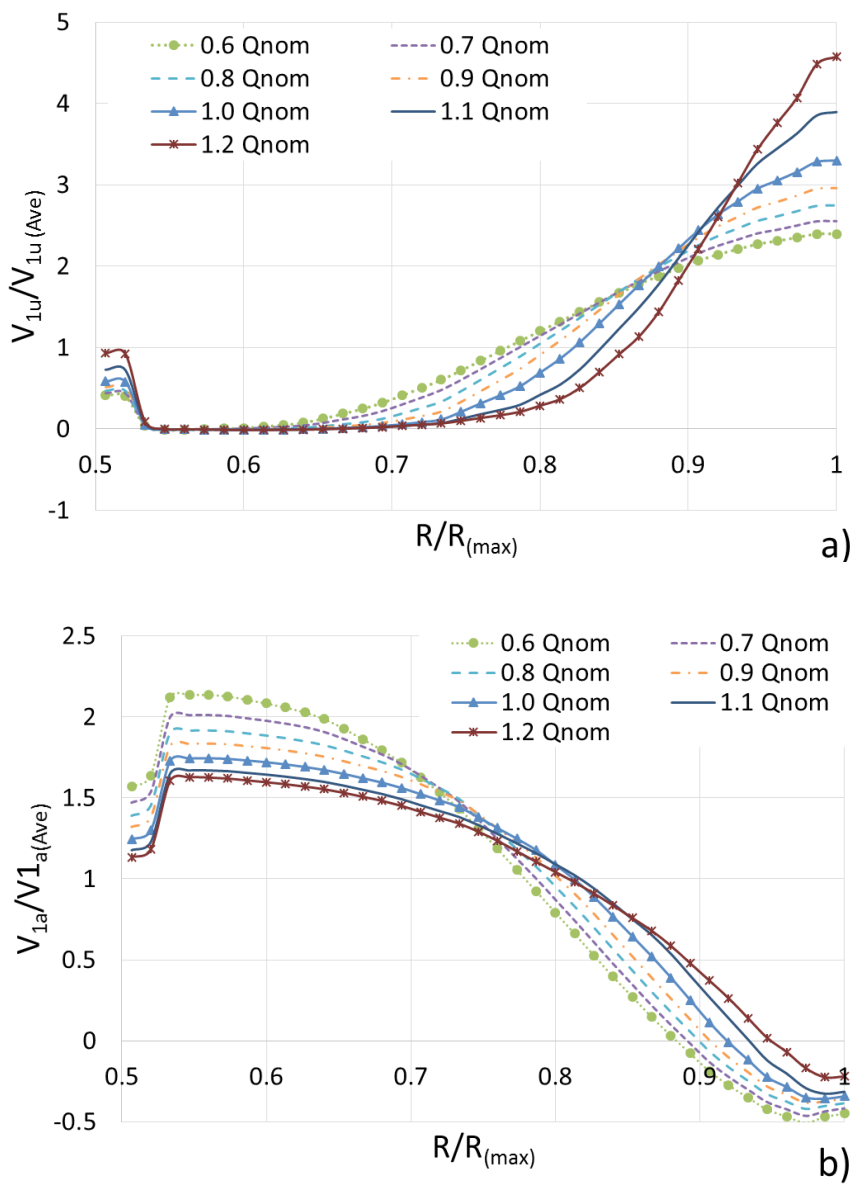

Fig. 9 Diagram of absolute velocity components distribution in cross section $B$ in the model №3 at flow rate range (0.6-1.2) $Q_{\text {nom }}$ :

a) Circumferential velocity component; b) Axial velocity component

The comparison of the diagrams of the circumferential and the axial components distribution of the absolute velocity in the cross section $B$ in the model №3 at flow range (0.6-1.2) $Q_{\text {nom }}$ indicate their dependence on flow changes in the value of the flow rate (Fig. 9). The increase of the relative circumferential component of the absolute velocity in the center of the cross-section of the impeller eye and the trend of decline in the periphery with a decrease in the value of the flow rate could be observed. This is the result of a reduction in the value of the absolute velocity axial component and consequently the increase of the stagnant zone.

In addition, the growing influence of the impeller (also described in $[8,11]$ ) and the effect of rotational walls of the axial inlet device forms the flow structure. The average circumferential component at $0.6 Q_{\text {nom }}$ and $1.2 Q_{\text {nom }}$ respectively is $24 \%$ bigger and $25 \%$ smaller than its value at $Q_{\text {пот }}$. Increasing the flow rate causes a decrease in the difference between the maximum and minimum values of the relative value of the axial component of the absolute velocity. Moreover, the intensity of return flow on the periphery of the impeller eye is reduced. The average value of the axial component at $0.6 Q_{\text {nom }}$ and $1.2 Q_{\text {nom }}$ respectively is $2.5 \%$ bigger and $2.9 \%$ smaller than its value for $Q_{\text {nom }}$. 


\section{Conclusion}

The results of the numerical simulation of the fluid flow in the double entry centrifugal pump are presented in this article. Four models of the axial inlet device with rotation and motionless inlet walls at the range of the flow rate $(0.6-1.2) Q_{\text {nom }}$ have been analyzed.

The change of the geometric dimensions of the diffuser section and the fairing at the models №1, №2 and №3 does not qualitatively affect the distribution of the components of the absolute velocity. Lengthening of the diffuser section and the fairing for $25 \%$ and $37 \%$ respectively in model №2 has led to the increase of the average circumferential component of absolute velocity by $2.7 \%$ while its axial component is constant. The use of cone fairing model №3 causes decrease of the average value of the angular and the axial components of the absolute velocity by $4 \%$ and $0.5 \%$ respectively. The analysis of the model №4 with the sudden expansion at the outlet of the diffuser section shows changing in the distribution of the circumferential component of the absolute velocity, which increases in the periphery, but decreases in the central part of its cross section. As a result, its average value is decreased by $19 \%$.

The rotation of the axial inlet device walls influences the change in the distribution of the axial component of the absolute velocity more than on circumferential component. The impeller causes growth of the circumferential component of the absolute velocity at the outlet of the diffuser section in the zone within (0.9-1.0) $D_{0}$. The backflow in the impeller inlet and the design features of the diffuser section and the fairing influence on the formation of an uneven distribution of the axial component of the absolute velocity. Changes of the average circumferential component of absolute velocity in average are $5.8 \%$ and axial $-1.7 \%$, in comparison with the condition of non-rotation of walls.

The reduction of the flow rate at the range from $1.2 Q_{\text {nom }}$ to $0.6 Q_{\text {nom }}$ increases the stagnant zone with the rotating motion of the liquid flow at the outlet of the diffuser section (in the case with rotation inlet walls). Average value of the circumferential component of the absolute velocity at the flow rate $0.6 Q_{\text {nom }}$ and $1.2 Q_{\text {nоm }}$ respectively is $24 \%$ bigger and $25 \%$ smaller than its value at the flow rate equaling $Q_{\text {nom }}$.

The design of model №4 has the greatest impact on the distribution of flow velocities at the impeller inlet. It has the highest rate of twist. However, the introduction of sudden expansion reduces the average value of the circumferential component and improves the distribution of the axial component of the absolute velocity.

\section{References}

[1] Gülich, J. F. "Centrifugal Pumps." 3rd ed., Springer, Springer, Berlin, Heidelberg. 2014.

https://doi.org/10.1007/978-3-642-40114-5

[2] Karapuzova, M., Lugova, S., Tverdokhleb, I. "Flow Structure Investigation in the Lateral Inlet Branches of Hydraulic Machines and Some Recommendations on Their Designing." Procedia Engineering. 39, pp. 140-147. 2012. https://doi.org/10.1016/j.proeng.2012.07.018

[3] Elin, A. V., Kochevsky, A. N., Konshin, V. N., Olsztynsky, P. L., Lugovaya, S. O., Schelyaev, A. E. "Testing the Package CFX-5 on Examples of Air Flow in the Elements of Flow Parts of Pumps of JSC VNIIAEN Specialization. Part 1. Simulation of Air Flow at a Lateral Combined Inlet of Diagonal Pump." Pumps \& Equipment. 1(36), pp. 20-24. 2006.

[4] Lipej, A., Mitruševski D. "Numerical Prediction of Inlet Recirculation in Pumps." International Journal of Fluid Machinery and Systems. 9(3), pp. 277-286. 2016

https://doi.org/10.5293/IJFMS.2016.9.3.277

[5] Song, X., Wood, H. G., Allaire, P. E., Antaki, J. F., Olsen D. B. "Inlet and Outlet Devices for Rotary Blood Pumps." Artificial Organs. 28(10), pp. 911-915. 2004.

https://doi.org/10.1111/j.1525-1594.2004.07399.x

[6] Rudolf, P., Klas, R. "Numerical simulation of pump-intake vortices." EPJ Web of Conferences. 92, 2015.

https://doi.org/10.1051/epjconf/20159202077

[7] Stepanoff, A. "Centrifugal and axial flow pumps: theory, design and application." 2nd ed., Wiley, New York. 1957.

[8] Ji, J. J., Luo X. W., Wu, Q. Y. "Design optimization of flow channel and performance analysis for a new-type centrifugal blood pump." IOP Conference Series: Materials Science and Engineering. 52(2), pp. 022012. 2013. https://doi.org/10.1088/1757-899X/52/2/022012

[9] Si, Q., Yuan, S., Yuan J., Bois, G. "Investigation on the influence of jetting equipment on the characteristics of centrifugal pump." Advances in Mechanical Engineering. 8(8), pp. 1-11. 2016. https://doi.org/10.1177/1687814016660287

[10] Cheah, K. W., Lee T. S., Winoto S. H. "Numerical Study of Inlet and Impeller Flow Structures in Centrifugal Pump at Design and Off-design Points." International Journal of Fluid Machinery and Systems. 4(1), pp. 25-32. 2011.

https://doi.org/10.5293/IJFMS.2011.4.1.025

[11] Mahaffey, R. M., van Vuuren, S. J. "Review of pump suction reducer selection: Eccentric or concentric reducers." Journal of the South African Institution of Civil Engineering. 56(3), pp. 65-76. 2014. [Online]. available from: http://www.scielo.org.za/pdf/jsaice/v56n3/08.pdf [Accessed: 10th May 2018]

[12] Zhang, Y., Luo, X., Yi, Y., Zhuang, B., Xu, H. "Investigation on the Flow Field Upstream of a Centrifugal Pump Impeller." International Journal of Fluid Machinery and Systems. 4(1), pp. 209-216. 2011. https://doi.org/10.5293/IJFMS.2011.4.1.209

[13] Lei, T., ShuLiang, C., ShaoBo, G. "Hydraulic design and pre-whirl regulation law of inlet guide vane for centrifugal pump." Science China Technological Sciences. 53(8), pp. 2142-2151. 2010. https://doi.org/10.1007/s11431-010-4005-5

[14] Rosa, S., Pinho, F. T. "Pressure drop coefficient of laminar Newtonian flow in axisymmetric diffusers." International Journal of Heat and Fluid Flow. 27, pp. 319-328. 2006.

https://doi.org/10.1016/j.ijheatfluidflow.2005.09.003

[15] Csizmadia, P., Hos, C. "CFD-based estimation and experiments on the loss coefficient for Bingham and power-law fluids through diffusers and elbows." Computers \& Fluids. 99, pp. 116-123. 2014. https://doi.org/10.1016/j.compfluid.2014.04.004 